\title{
Chromosome evolution at the origin of the ancestral vertebrate genome
}

\author{
Christine Sacerdot ${ }^{1}$, Alexandra Louis ${ }^{1}$, Céline Bon ${ }^{1,2}$, Camille Berthelot and Hugues Roest Crollius ${ }^{1 *}$ (D)
}

\begin{abstract}
Background: It has been proposed that more than 450 million years ago, two successive whole genome duplications took place in a marine chordate lineage before leading to the common ancestor of vertebrates. A precise reconstruction of these founding events would provide a framework to better understand the impact of these early whole genome duplications on extant vertebrates.

Results: We reconstruct the evolution of chromosomes at the beginning of vertebrate evolution. We first compare 61 extant animal genomes to reconstruct the highly contiguous order of genes in a 326-millionyear-old ancestral Amniota genome. In this genome, we establish a well-supported list of duplicated genes originating from the two whole genome duplications to identify tetrads of duplicated chromosomes. From this, we reconstruct a chronology in which a pre-vertebrate genome composed of 17 chromosomes duplicated to 34 chromosomes and was subject to seven chromosome fusions before duplicating again into 54 chromosomes. After the separation of the lineage of Gnathostomata (jawed vertebrates) from Cyclostomata (extant jawless fish), four more fusions took place to form the ancestral Euteleostomi (bony vertebrates) genome of 50 chromosomes.

Conclusions: These results firmly establish the occurrence of two whole genome duplications in the lineage that precedes the ancestor of vertebrates, resolving in particular the ambiguity raised by the analysis of the lamprey genome. This work provides a foundation for studying the evolution of vertebrate chromosomes from the standpoint of a common ancestor and particularly the pattern of duplicate gene retention and loss that resulted in the gene composition of extant vertebrate genomes.
\end{abstract}

\section{Background}

New gene copies largely appear by small-scale duplication during genome evolution [1], contributing to genetic innovation and phenotypic diversity [2]. In vertebrate evolution, whole genome duplications (WGDs) are rare, in contrast to plants where they appear to be more frequent [3]. More than 450 million years ago, an early vertebrate lineage was subject to two WGD in relatively rapid succession prior to its diversification into about 60,000 extant species (Fig. 1). Envisioned by Susumo Ohno since the early 1970s [4], these events known as the "1R-2R hypothesis" have since been firmly established by several genome-wide studies [5-7].

\footnotetext{
* Correspondence: hrc@ens.fr

'Institut de Biologie de l'Ecole Normale Supérieure (IBENS), Ecole Normale Supérieure, CNRS, INSERM, PSL Research University, 75005 Paris, France Full list of author information is available at the end of the article
}

Approximately $35 \%$ of extant human genes still exist in duplicate copies (ohnologs) owing to the 1R-2R WGDs [8, 9], including the four clusters of HOX genes found in all tetrapods [10]. Ohnologs represent the vast majority of duplicated genes in the human genome [11]. They have been shown to be enriched in disease genes $[8,12-14]$ and to influence the frequency of structural variations in human populations [15]. The ancient $1 \mathrm{R}-2 \mathrm{R}$ genome duplications therefore still exert a strong influence on present-day genomes, warranting a better understanding of their early history. The first reconstruction of the evolution of the karyotypes before, during, and after the 1R-2R [6] left a number of questions unanswered. It could not reliably identify all ancestral vertebrate chromosomes nor could it determine if chromosome fusions or fissions took place between the two WGDs. This low resolution was due to the many chromosome rearrangements that have occurred since

(c) The Author(s). 2018 Open Access This article is distributed under the terms of the Creative Commons Attribution 4.0 International License (http://creativecommons.org/licenses/by/4.0/), which permits unrestricted use, distribution, and 


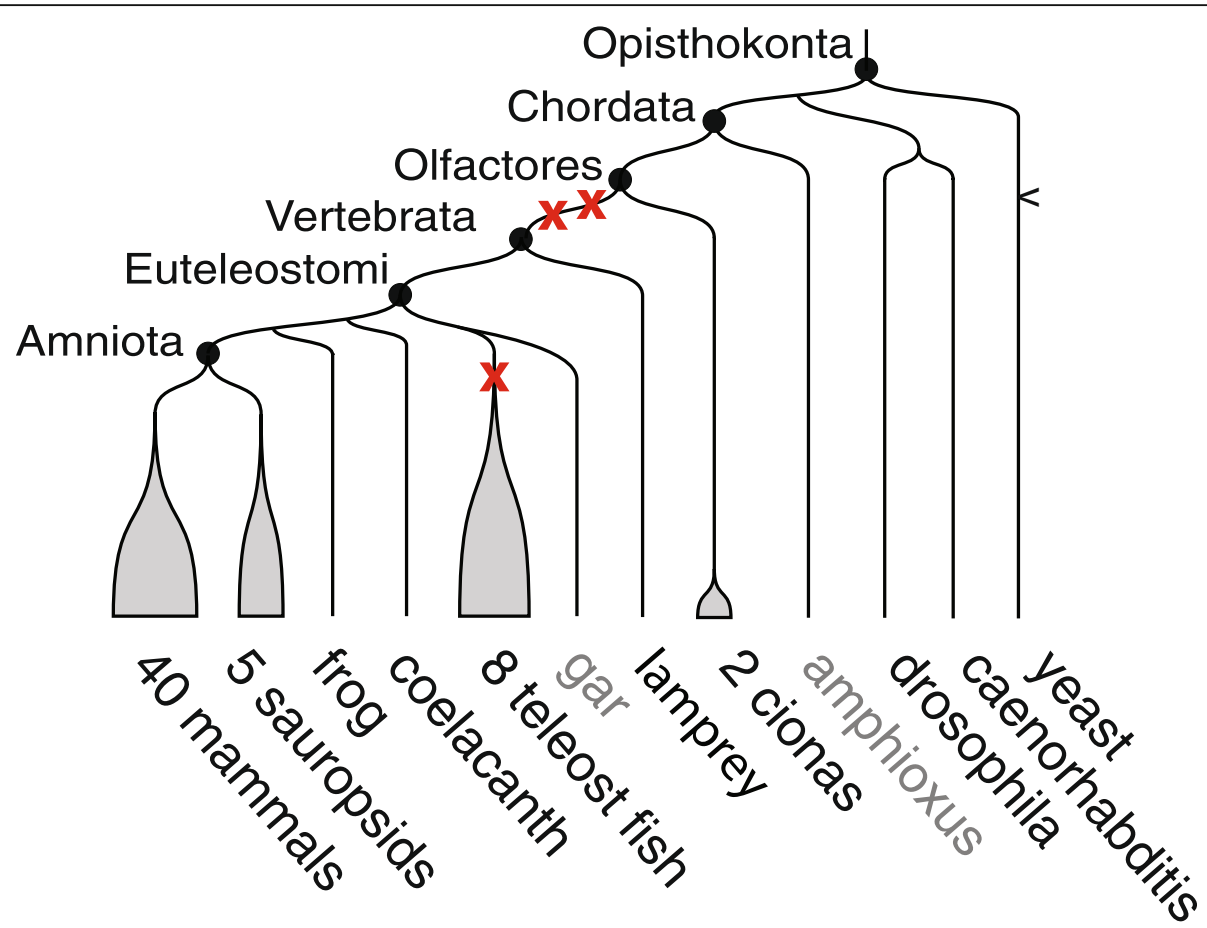

Fig. 1 Schematic phylogenetic relationships between species used in this study. Species or groups of species shown in black at the end of branches are the 61 species included in Ensembl release 69, and were used to reconstruct the ancestral Amniota genome. Sauropsids include birds and reptiles. Species shown in grey were used at other stages in the analysis. Black circles materialize ancestral genomes relevant to this study. Red crosses indicate the relative positions of WGDs: two before the vertebrate radiation and one before the teleost fish radiation. Branches are not to scale

the vertebrate ancestor (Vertebrata), scrambling the ancestral gene organization in extant genomes, and the absence of a suitable outgroup species. In addition, a recent comparison between the lamprey genome and the chicken genome questioned the $1 \mathrm{R}-2 \mathrm{R}$ hypothesis and suggested that a single WGD and multiple segmental duplications could also explain the synteny patterns observed $[16,17]$ between these two species. Both the uncertainties of the first reconstruction of the pre-1R genome and this alternative to the $1 \mathrm{R}-2 \mathrm{R}$ hypothesis motivate a detailed analysis of the early evolution of vertebrate chromosomes.

The number of sequenced vertebrate genomes has recently greatly increased, allowing the reconstruction of ancestral genomes with much higher accuracy. After reconstructing the ancestral Amniota genome as a stepping stone to better understand the details of early vertebrate karyotype evolution, we find that the pre-1R vertebrate genome contained 17 chromosomes and that all vertebrates descend from a post- $2 \mathrm{R}$ genome comprising 54 chromosomes. We show that the human genome still bears a strong imprint of the pre- $1 \mathrm{R}$ genome, and provide resources to study the impact of the two WGDs in vertebrates from the perspective of a reference point shared by all descendent species.

\section{Results}

Identification of pairs of ohnologous genes in the ancestral Amniota genome

We inferred from Ensembl gene trees (version 69) that 19,786 genes existed in the ancestral Amniota genome, the ancestor of birds, reptiles, and mammals (326 million years). We used AGORA (Algorithm for Gene Order Reconstruction in Ancestors) [18] to order and orient these genes as they were in the Amniota genome (Additional file 1: Figure S1). This in silico reconstruction is composed of 470 segments, with $50 \%$ of the genes in segments larger than 253 genes (N50 length). We then selected the 56 chromosome-size segments larger than 50 genes as an initial set of Contiguous Ancestral Regions (CARs; mean CAR length 256 genes, 12,134 genes total) to identify duplicated regions.

Ohnologs resulting from the $1 \mathrm{R}-2 \mathrm{R}$ WGDs are key to identifying pairs of duplicated chromosome segments. We identified pairs of putative ohnologous genes directly in the reconstructed Amniota genome using gene trees to date their duplication while ensuring that each member of a pair belongs to a different CAR (see the "Methods" section), resulting in a "List A" containing 5616 ancestral Amniota ohnolog pairs. Two previous studies have also identified ohnologs from the two 
WGDs, in the human and other vertebrate genomes, using conserved synteny and sequence similarity. We used Ensembl gene trees to convert extant gene identifiers from these studies to their ancestral Amniota gene identifiers. The first list established by Makino and McLysaght [8] and hereafter called "List B" contains 4870 ancestral Amniota gene pairs. The second study by Singh et al. [9] established three levels of confidence (strict, intermediate, and relaxed) to define ohnologs. Following these criteria, we defined three additional lists containing respectively 2873 (List "C-strict"), 5253 (List "C-inter"), and 7806 (List "C-relax") ancestral Amniota ohnolog pairs.

The sum of the A, B, and C-relax lists shows only $25 \%$ of genes in common (Fig. 2a), but we show below that the three lists nevertheless support the $1 \mathrm{R}-2 \mathrm{R}$ hypothesis. In this scenario, each original chromosome is duplicated in two then four copies; chromosomes thus form tetrads where each possesses three ohnologous counterparts. We tested whether pairs of CARs share more ohnologs than expected if they were distributed randomly using each of the five lists of ohnologs (proportionality test; see the "Methods" section). We show that, in all cases, CARs are ohnologous to three other CARS on average (Additional file 1: Figure S2). Despite their differences, all lists therefore support the 1R-2R hypothesis, justifying the construction of an improved consensus list of ancestral Amniota ohnolog pairs using all five lists. We started from the intersection of lists A, B, and $\mathrm{C}$-strict as the most reliable subset (1273 pairs of ohnologs) and gradually extended it by adding pairs of genes from lower confidence subsets (pairs of genes intersecting fewer lists, or lists that include $\mathrm{C}$-inter and C-relaxed; Fig. 2b). In this process, we ensured that the
A

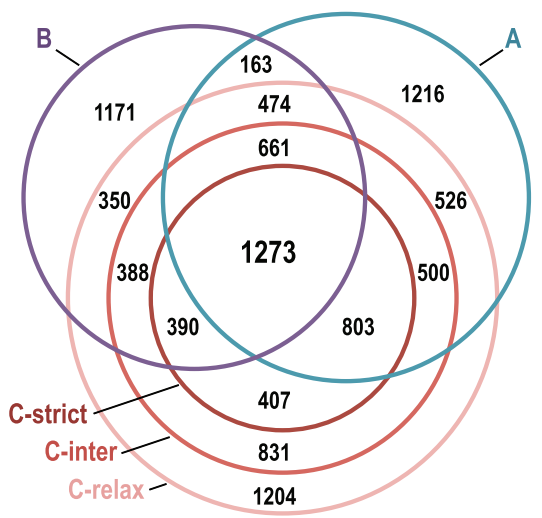

B

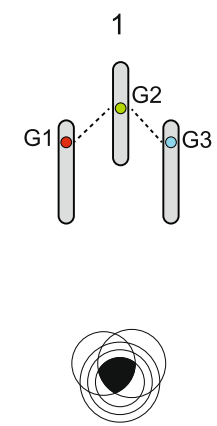

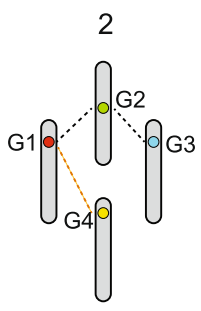

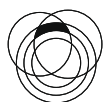

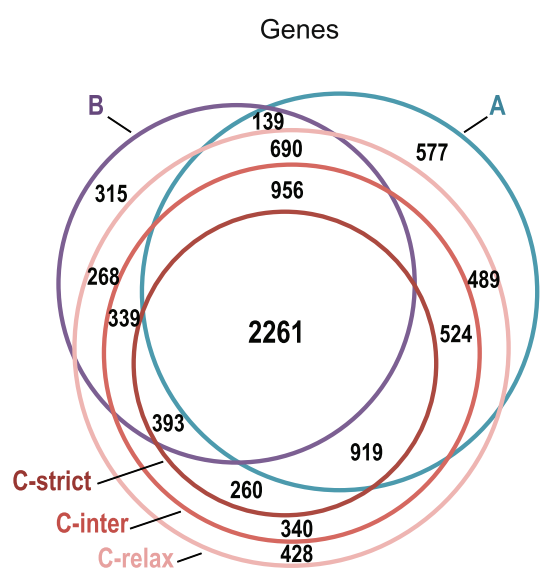

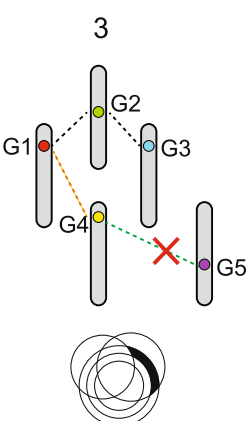

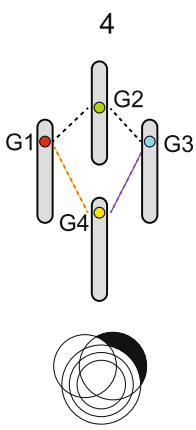

Fig. 2 Identification of ohnolog pairs in the ancestral Amniota genome. (a) Comparison between five lists of ohnolog pairs in Amniota. Left: a Venn diagram of the sets of ohnolog pairs from five lists: list A (this study), list B [8] and the three lists C [9]. The numbers of pairs at the intersections of the lists are indicated. Right: a Venn diagram of the sets of ohnolog genes from the same lists as above. The overlap between the lists of ohnolog genes is higher than between the lists of pairs because the latter contain different pairs between the same genes. For example, two pairs G1-G2 and G1-G4 are in different lists (no overlap between lists) but gene G1 is common to both lists (1 gene overlap; see (b) for a graphical illustration). The surface of the circles and their intersection are roughly proportional to the number of genes pairs or genes of each list. (b) Schematic example of ohnolog pair selection. Step 1: from the initial list of 1273 gene pairs (black area in Venn diagram), 2 pairs involve 3 genes G1, G2, and G3, each on a different CAR. Step 2: pairs from a new sub-list are considered, a new gene pair G1-G4 is added to the network. Gene G4 is on a fourth CAR. Step 3: A new list is considered, a new pair is identified (G4-G5) but G5 is on a fifth CAR so pair G4-G5 is discarded. Step 4: a new list is considered, a pair G4-G3 supporting the network is identified 
growing list remained compatible with the $1 \mathrm{R}-2 \mathrm{R}$ hypothesis: a pair could never be included if the two genes belong to two different phylogenetic trees (i.e., the two duplicated genes in a pair must descend from a common ancestral gene) and all the ohnologs within a phylogenetic tree, when arranged in pairs, cannot link more than four CARs (a tetrad; Fig. 2b and Additional file 1). This incremental process (Additional file 2: Table S5) resulted in a list of 8184 ohnologous genes, forming 7441 ohnolog pairs grouped into 2973 ohnolog families, each family in principle corresponding to one pre-1R gene (Additional file 3, 4, and 5 respectively for the list of ohnolog genes along with their human descendants, the list of ohnolog pairs and the list of ohnolog families).

This list of ohnolog pairs is of high quality for a number of reasons. First, it is based on the gene content and synteny in the reconstructed ancestral Amniota genome which is 326 million years closer to the $1 \mathrm{R}-2 \mathrm{R}$ events than extant genomes, and thus, signatures of $1 R-2 R$ events are read with greater accuracy. Second, this list abides by a $1 \mathrm{R}-2 \mathrm{R}$-compatibility rule, i.e., no ohnologous gene family connects more than four CARs. Third, the ohnologous pairs are all phylogenetically consistent in that both genes in a pair always belong to the same Ensembl gene tree. Fourth, the two genes of a pair were allowed to be on the same CAR only if $\geq 90$ genes separated them to avoid spurious inclusions of genes duplicated in tandem.

\section{Identification of post-2R duplicated CARs}

Using our improved list of ohnolog pairs, we manually split, assembled, and grouped ancestral Amniota CARs in order to convert them to a configuration that is as close as possible to the post-2R karyotype. In the simplest scenario, post-2R CARs should readily form tetrads of four ohnologous CARs, each corresponding to one pre-1R chromosome. However, chromosome rearrangements between the $1 R$ and $2 R$, between the $2 R$ and Amniota, and incomplete or incorrect reconstruction of CARs all concur to disrupting this ideal pattern. We started with the 56 largest CARs and applied the proportion test to identify CARs sharing a significant number of ohnologous genes as described above (i.e., ohnologous CARs). We identified groups of at least three CARs all significantly ohnologous pairwise ( $p$ value $<5.10^{-2}$, Bonferroni adjusted). These were completed into tetrads (i.e., four CARs all significantly ohnologous to each other) by including smaller CARs and/or CARs at lower significance thresholds. We also merged CARs that showed evidence of belonging to the same Amniota chromosome (Table 1), because they were merged in alternative AGORA reconstructions using different sets of parameters, and/or they showed identical homologies to Amniota descendent genomes (human or chicken; Fig. 1).
In addition, merged CARs had to be significantly ohnologous to at least one CAR in common in a triad and show no significant ohnology with each other. We also split CARs that showed, along their length, a disruption in their distribution of ohnologs and disruption of their homologies to chicken, human, spotted gar, or medaka chromosomes (Fig. 1). Finally, we confirmed merged CARs using homologies with outgroup species such as the spotted gar or the medaka (Fig. 1; see the "Methods" section).

The resolution of CARs in tetrads is much clearer after this conversion of Amniota CARs to a post-2R configuration, especially compared to human chromosomes (Fig. 3a). The proportionality test links each curated Amniota CAR on average to 3 other CARs almost independently of any $p$ value threshold, whereas human chromosomes are much more sensitive to the $p$ value threshold. Indeed in the human genome, the expected average of three partners per chromosome is reached only at a $p$ value of $1.10^{-09}$ (Fig. $3 \mathrm{~b}$ ), a stringent threshold where 7 human chromosomes cannot be assigned to a tetrad. An example of construction of a tetrad and assembly of CARs is detailed in Fig. 3c. We identified three significantly ohnologous CARs grouped in a triad (CARs 73, 117, 250). Two additional CARs (256 and 137) show significant ohnology to CAR 250. The tetrad was completed with the addition of a smaller CAR (CAR 82), which was linked to four of the five initial CARs with significant but higher $p$ values (Additional file 6). Then, of the five initial CARs, three fulfilled the conditions to be assembled in a single larger CAR (CARs 117, 137, 256): they were ohnologous to CARs in common but were not ohnologous to each other, and AGORA merged CARs 117/137 and CARs 137/256 together when using a more recent version of Ensembl (Version 84). Furthermore, the three CARs map to the same chicken and spotted gar chromosomes, strongly suggesting that they derive from the same chromosome of their common Vertebrata ancestor. Finally, all three assembled CARs, when mapped on the medaka genome (a teleost fish that went through an additional WGD [19]), are orthologous to the same two medaka chromosomes (13 and 14; Additional file 6).

We identified two post-2R chromosomal fusions that required a split of two Amniota CARs in two sub-CARs each (CARs 5 and 118), and we identified three probable assembly errors that required a split of three CARs (CARs 40, 46, and 97). We also performed 23 CAR assemblies (Table 1), ending with a final set of 51 CARs edited to more closely represent their post- $2 \mathrm{R}$ configuration. The ohnology relationships between CARs based on significant $p$ values of the proportion test connected the 51 CARs into 17 tetrads (Fig. 3d). The precise step-by-step procedure that we followed to split or assemble CARs and group 
Table 1 Assembly of Amniota CARs

\begin{tabular}{|c|c|c|c|c|c|c|}
\hline Assembled CARs & $\begin{array}{c}\text { AGORA } \\
\text { alternative }\end{array}$ & $\begin{array}{c}\text { Ensembl } \\
\text { v. } 84\end{array}$ & $\begin{array}{c}\text { Chicken }(+) \\
\text { or human }(x) \\
\text { synteny }\end{array}$ & $\begin{array}{l}\text { Spotted } \\
\text { gar } \\
\text { synteny }\end{array}$ & $\begin{array}{c}\text { Gar- } \\
\text { chicken } \\
1: 1\end{array}$ & $\begin{array}{c}\text { Double } \\
\text { conserved } \\
\text { synteny } \\
\text { medaka } \\
\end{array}$ \\
\hline $6,39,140$ & + & - & + & + & & + \\
\hline 80,120 & + & + & + & + & & + \\
\hline 107,207 & + & - & + & + & + & + \\
\hline $5 a, 152$ & - & - & + & + & & + \\
\hline 254,48 & - & - & $x$ & n.d. & & + \\
\hline $65,53,103$ & + & - & + & + & & + \\
\hline 114,195 & + & + & + & + & + & + \\
\hline 26,102 & + & + & + & + & + & + \\
\hline $50,85,84$ & - & + & $x$ & + & & + \\
\hline $67,111,83$ & $67 \& 83$ & - & + & + & + & + \\
\hline $146,450,456,393,52$ & - & $146 \& 450$ & $x$ & n.d. & & $146,393 \& 52$ \\
\hline $117,137,256$ & $117 \& 137$ & $137 \& 256$ & + & + & + & + \\
\hline $204,75,55$ & $204 \& 75$ & - & + & + & & + \\
\hline $129,261,97 a$ & $261 \& 97$ & - & + & + & & + \\
\hline 59,420 & & - & $x$ & + & & + \\
\hline 3,22 & + & + & + & + & + & + \\
\hline 60,148 & + & + & + & + & & + \\
\hline $10,240,2$ & $10 \& 240$ & - & + & + & & + \\
\hline $5 b, 40 b$ & - & + & + & + & & + \\
\hline $118 a, 192,14,172$ & $14 \& 192$ & - & + & + & & + \\
\hline $66,46 a$ & - & - & $x$ & + & & + \\
\hline $123,46 b$ & + & + & + & + & & + \\
\hline $441,430,93$ & - & + & $x$ & n.d. & & + \\
\hline
\end{tabular}

Each line corresponds to the assembly of two to five CARs (column 1). "AGORA alternative" indicates that all CARs (+) or some CARs (CAR number shown) are merged as a single CAR in alternative AGORA reconstructions with different parameters. "Ensembl v.84" indicates that all CARS (+) or some CARs (CAR number shown) are merged in an AGORA reconstruction of the Amniota genome based on a more recent version of Ensembl (v. 84). "Chicken or human synteny" indicates that all assembled CARs are homologous to the same chicken (+) or human (x) chromosome. "Spotted gar synteny" indicates that assembled CARs are homologous to the same spotted GAR chromosome. Gar-chicken 1:1 indicates that assembled CARs are homologous to the same gar and chicken chromosomes. Double conserved synteny with medaka indicates that the assembled CARs are syntenic with the same pairs of chromosomes in the medaka genome. Greyed columns indicate that at least one of these conditions must be fulfilled for assembling CARs. Non-greyed columns are supporting evidence but dispensable conditions. A full description of the table can be found in Additional file 1

them in tetrads is detailed in Additional file 1, including Additional file 1: Figure S7B to S14B.

\section{Chromosome evolution between the $1 \mathrm{R}$ and $2 \mathrm{R}$ whole genome duplications}

The 17 tetrads composed of 51 Amniota CARs are not all disjoint: some share one or two CARs in common, reflecting chromosomal events between the $1 \mathrm{R}$ and the $2 \mathrm{R}$, and after the $2 \mathrm{R}$. We identified these events by first establishing theoretical scenarios corresponding to each configuration. A single disjoint tetrad implies a simple evolutionary scenario without any large chromosomal rearrangement between the two WGDs (Fig. 4a). Two adjacent tetrads, however, can be explained by one of two scenarios, each with the same degree of parsimony: a post-1R chromosome descending from a single pre-1R chromosome was broken (a fission), or two post-1R chromosomes, each descending from separate pre-1R chromosomes, were merged (a fusion; Fig. 4b). As previously noted [20,21], a non-duplicated outgroup species would be helpful to discriminate between the two possible ancestral configurations. However, of the two nearest outgroups to vertebrates (Fig. 1), neither tunicates (e.g., species of the Ciona group) nor cephalochordates (e.g., the amphioxus Branchiostoma floridae) [7, 22] are suitable for this purpose. The former are too diverged to identify clear chromosome homologies, and the genome of $B$. floridae is too fragmented to be informative. To circumvent this problem, we used a previously published reconstruction of 17 Chordate Linkage Groups (CLG) 
A
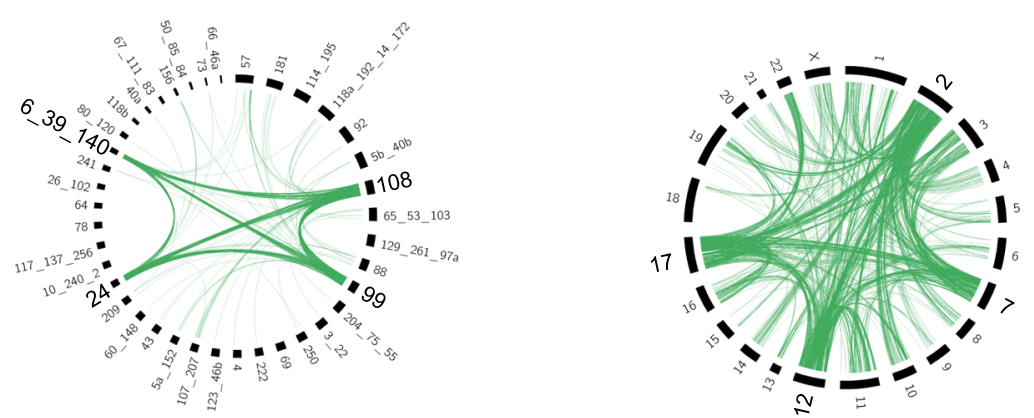

B
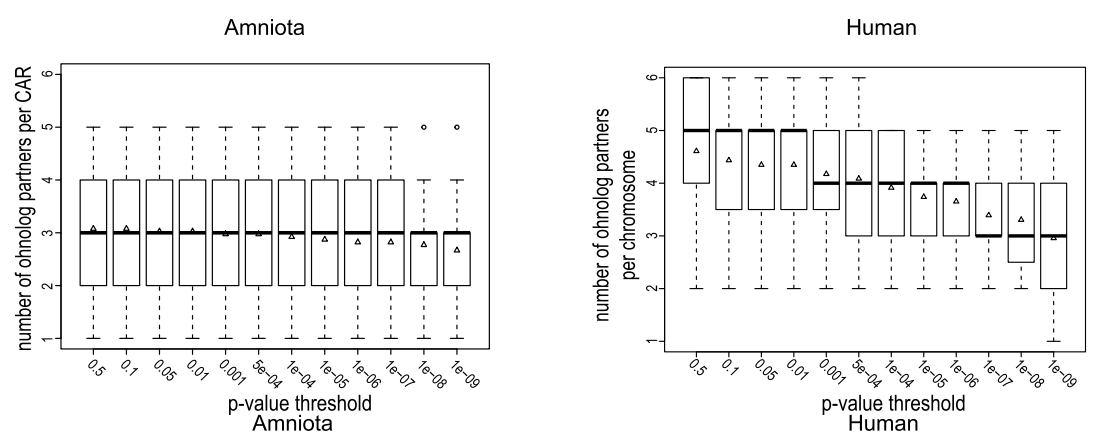

C

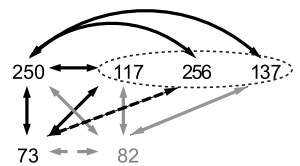

D
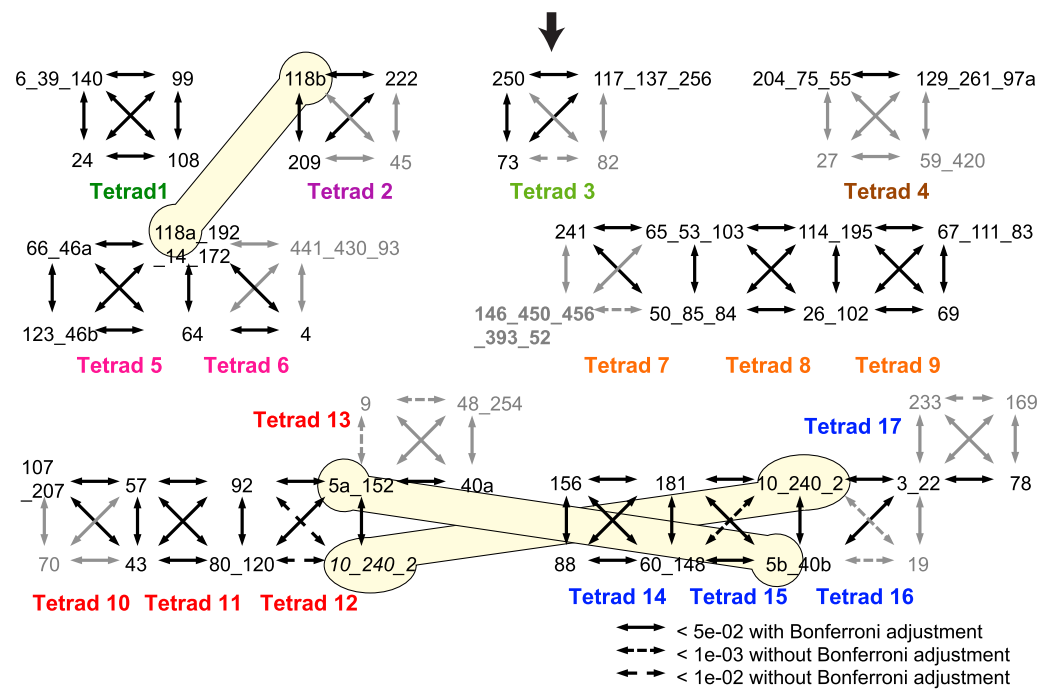

Fig. 3 (See legend on next page.) 
(See figure on previous page.)

Fig. 3 Organization of ancestral Amniota CARs in tetrads (a) Circos plot [45] showing the pairs of ohnologs involving each of the four chromosomes (Homo sapiens) or CARs (Amniota) of the tetrad carrying the Hox genes (Tetrad 1 in D). The pairs of ohnologs in the human genome were the descendants of those of Amniota (6121 pairs of human ohnologs vs. 7441 pairs of amniote ohnologs). The human Hox cluster tetrad is mainly composed of human chromosomes 2, 7, 12, and 17. The Amniota Hox cluster tetrad is composed of CARs 108, 24, 99, and 6_39_140. An ohnolog pair is represented (green lines) between two Amniota CARs or two human chromosomes if at least one of the two genes of the pair falls on a chromosome/CAR of the tetrad. The Amniota Hox CARs are involved in 634 pairs, while the human Hox chromosomes are involved in 2171 pairs of ohnologs. This figure shows that the reconstruction of Amniota ancestor displays a clearer picture of the 1R-2R than the human genome. (b) Ohnolog partners per CAR/chromosome in the Amniota (left) and human (right) genomes. Each boxplot shows the distribution of the number of CARs (Amniota) or chromosomes (Human) found to be ohnologous to a given CAR/chromosome by the proportionality test. The $x$-axis shows the Bonferroni adjusted $p$ value thresholds used to select ohnologous chromosome/CARs. Triangles indicate the average number of partners. The Amniota genome shows a clear and stable distribution of three partners per CAR across a wide range of $p$ values, as expected after two WGDs where chromosomes are grouped in tetrads. In contrast, the distribution in Homo sapiens shows that extremely low $p$ value thresholds must be used to reach the expected average of three partners, justifying the fragmentation of the human genome as described in [6]. (c) Example of how a group of significantly ohnologous CARs was analyzed to form tetrad 3. Black double-headed arrows ( $p$ value $<5.10^{-2}$ after Bonferroni adjustment) represent the raw output of the proportion test, showing CARs with significant ohnology relationships. CARs 73, 117, and 250 form a triad of mutually ohnologous CARs. Dotted lines are additional ohnologous relationships that are supported without the Bonferroni adjustment. Numbers in black indicate CARs of at least 50 genes, while smaller CARs ( $<50$ genes) are in grey. Additional evidence (see text) was used to complete the tetrad. (d) Seventeen tetrads composed of 51 CARs. CARs are numbered arbitrarily and are joined by underscores in an arbitrary order when assembled. The letters "a" or "b" indicate that the CAR has been split in two segments (CARs 5 and 118) as part of the conversion to a post-2R karyotype (see text) and one CAR is present twice in two different tetrads (CAR 10_240_2) to facilitate the representation (pale yellow shapes)

A A cluster with one tetrad

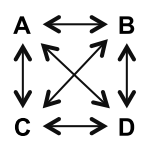

B A cluster with two adjacent tetrads

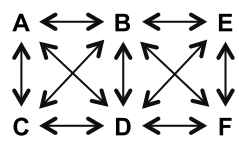

Evolutionary scenario

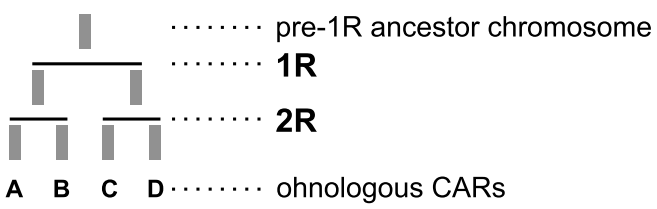

Fission scenario

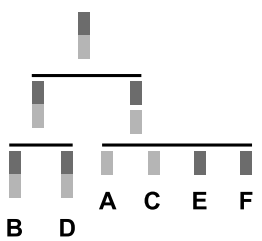

Fusion scenario

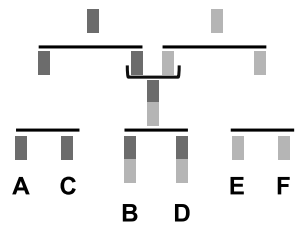

C Two tetrads with one CAR in common
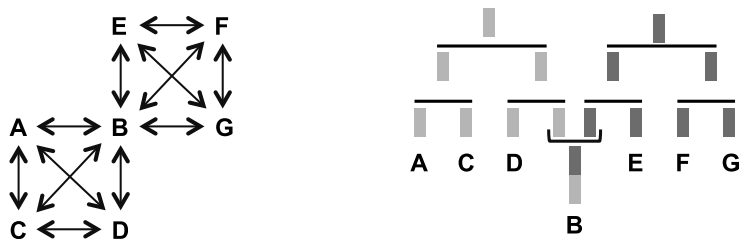

Fig. 4 Evolutionary scenario models. (a) A single evolutionary scenario explains the formation of a single disjoint tetrad of ohnologous CARs. (b) Two equally possible evolutionary scenarios can explain how ohnologous CARs can form two adjacent tetrads: a fission or a fusion of chromosomes could have occurred between the 2 WGDs. In each case, the B and D chromosomes each possess two distinct parts (dark and light grey) homologous to distinct chromosome sets. B and D are therefore common to two tetrads. (c) A chromosome fusion after the two WGDs explains how two tetrads can be joined via a single CAR 
(Additional file 1: Figure S15), which are groups of human genes descended from the same ancestral chordate chromosome [7]. This reconstructed proto-karyotype precedes the $1 \mathrm{R}-2 \mathrm{R}$ events by less than 50 million years and is located at a much shorter evolutionary distance to the Vertebrata ancestor than the extant amphioxus genome (Fig. 1).

Remarkably, each CLG was associated with one predominant CAR tetrad (Additional file 1: Figure S16). Consequently, all seven adjacent tetrads result from chromosome fusions between the $1 \mathrm{R}$ and $2 \mathrm{R}$ WGDs. Indeed, a chromosome fission would have split the gene content of a CLG over two different tetrads (Fig. 4b). Incidentally, we also found evidence for a chromosome fusion between the chordate ancestor and the $1 \mathrm{R}$, because more than 75\% of genes from CLGs 6 and 7 have their descendants in a single tetrad (tetrad 14; Additional file 1: Figure S16 and Additional file 7). Conversely, we could not confidently assign tetrad 13 to a CLG, likely because it is a small tetrad. We therefore conclude that the pre-1R karyotype comprised 17 chromosomes, duplicated into 34 chromosomes after the first WGD and followed by seven fusions. The resulting 27 chromosomes were duplicated in the second WGD leading to 54 Vertebrata chromosomes, at the origin of the approximately 60,000 extant species of vertebrates.

\section{Chromosome evolution after the $2 \mathrm{R}$}

This karyotype was followed by additional chromosome fusions at different stages after the 2R WGD. Four fusions followed the scenario described in Fig. 4c, where a single CAR joins two tetrads (CAR 5, CAR5a_152, CAR3_22, CAR 10_240_2; Fig. 3d). They can be dated to the period between the 2R WGD and the Euteleostomi ancestor because of their homologies to both descendent and outgroup genomes. For example, CAR_10_240_2 is homologous to a single chicken chromosome (GG4), a single human chromosome (chromosome $\mathrm{X}$ ) and a single spotted gar chromosome (LG7), which is most parsimoniously consistent with a situation where this CAR was already a single chromosome in Euteleostomi, the common ancestor of these three species. A fifth fusion can be dated to the period between Euteleostomi and Amniota: CAR 118 is common to two tetrads but while it is homologous to a single chicken chromosome (GG1), a disruption of synteny in the spotted gar genome and a disruption in the DCS pattern in the medaka genome are consistent with a fusion in the lineage leading to Amniota.

Accounting for these fusions, the 54 chromosomes in the post-2R Vertebrata led to a Eueteleostomi karyotype of 50 chromosomes (4 fusions) and to an Amniota karyotype of 49 chromosomes (1 fusion; Fig. 5 and Additional file 1). A dedicated Genomicus server [23] provides a graphical

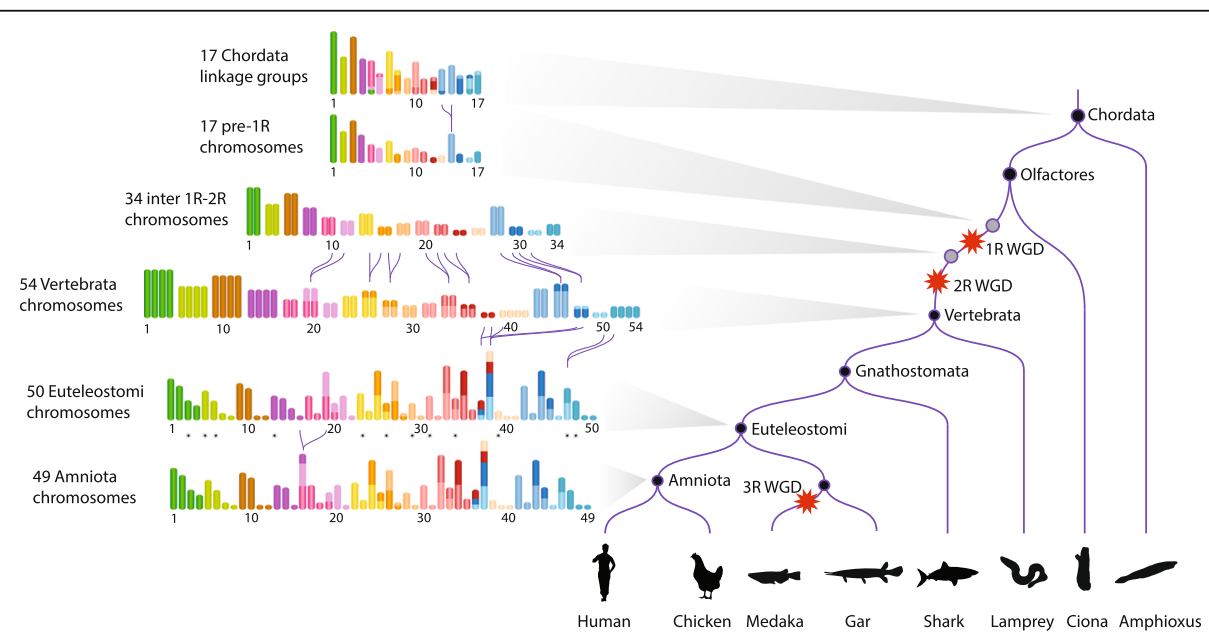

Fig. 5 Reconstructed evolutionary history of karyotypes from Chordata to Amniota. On the right, a simplified species tree of the Chordata is shown, with WGD events depicted by red stars. The eight lineages represented from left to right are mammals, birds, teleost fish, holostocean fish (gar), cartilaginous fish, cyclostomes (lamprey, hagfish), tunicates (ciona), and cephalochordates (amphioxus). On the left, successive reconstructed karyotypes are shown, with one color for each of the 17 pre-1R chromosomes. The length of each pre-1R chromosome is proportional to its number of genes. For the 17 Chordate Linkage Groups (CLGs) of [7], the size of the colored segment is proportional to the number of genes that are found in the intersection of the CLG with a pre-1R chromosome, although segments corresponding to $<10 \%$ of the number of genes of the CLG were omitted for clarity (Additional file 1: Table S7). The karyotype between $1 R$ and 2R was deduced from the pre$1 R$ karyotype and the seven chromosome fusions are shown with purple curvy lines joining the fused chromosomes. The Euteleostomi karyotype was deduced from the Vertebrata karyotype after four chromosome fusions (Additional file 1). The lengths of the Euteleostomi chromosomes are proportional to the number of genes in the homologous Amniota CARs. Finally, the Amniota karyotype differs from that of Euteleostomi by only one chromosome fusion. The Amniota chromosomes were numbered from 1 to 49 (Additional file 1: Table S11 for correspondence with the CARs and number of genes). Black stars under 12 Euteleostomi chromosomes denote predicted ancestral micro-chromosomes 
interface to compare and analyze the genomes presented in this work (Additional file 1: Figure S17; http://genomicus.biologie.ens.fr/genomicus-69.10/).

\section{Comparative genomics between the pre-1R genome and the human genome}

To enable comparisons between the pre-1R vertebrate ancestor genome reconstruction and extant species, we assigned genes to each of the 17 pre-1R chromosomes. Among Amniota genes, only ohnologs could be confidently assigned to pre-1R chromosomes, as non-ohnolog genes could have been acquired after the $1 \mathrm{R}$ WGD. To circumvent this problem, we applied a conservative procedure to assign 5052 of the 10,093 ancestral Olfactores genes to the 17 predicted pre-1R chromosomes (Additional file 8). The Olfactores ancestor is the common ancestor of vertebrates and tunicates and the closest ancestor upstream of the reconstructed pre-1R genome in Ensembl gene trees. This set of ancestral genes provides a direct connection to the human genome, through their 8378 human descendent genes. By identifying each of these human descendants by the color of its ancestral pre-1R chromosome (Fig. 6), we show that the structure of the 17 pre-1R chromosomes is still strikingly apparent in the human genome, with some chromosomes almost entirely composed of genes from a single pre-1R chromosome (e.g., chromosomes 14 and 15). We measured the degree of conservation of the post-1R-2R ohnolog content in windows of 50 genes positioned every 10 genes across human chromosomes. Three regions overlapping the Hox clusters A, $\mathrm{B}$, and $\mathrm{D}$ stand out (and Hox $\mathrm{C}$ to a lesser degree; Fig. 6), in line with the known functional importance associated with the clustering of these ohnologs [24].

The four Hox clusters originate from pre-1R chromosome 1, and we examined in the same light other paralogous clusters that have been proposed to originate from a single pre- $1 \mathrm{R}$ locus. The $\mathrm{MHC}$ region on human chromosome 6 contains a number of genes unrelated to immune functions but which possess ohnologs in 3 other loci on chromosomes 1, 9, and 19 [25]. All 4 regions descend from pre-1R chromosome 9. Similarly, the loci containing FOX gene clusters have been compared and found to share paralogs suggestive of en-bloc duplications early in vertebrate evolution [26]. We confirm here their unique origin on pre-1R chromosome 10 . In contrast, no common origin was found for the different clusters of imprinted genes in the human genome (e.g., H19 locus on chromosome 11, IGF2R locus on chromosome 6, PON [1-3] locus on chromosome 7, UBE3A locus on chromosome 15) [27, 28], in line with their known progressive appearance later in therian mammals [29]. The evolutionary scenario of ancestral vertebrate chromosomes presented here is therefore consistent with our current view of the evolution of these important gene families.

Finally, we analyzed the frequency of Gene Ontology terms of the human descendants of the 1416 pairs (2 gene losses), 502 triplets (1 gene loss), and 172 quartets (no loss) of ancestral Amniota ohnologs and find a striking pattern: quartets are enriched in both neuronal development and neuronal function (synaptic transmission) and triplets are enriched in muscle development (especially heart) and in muscle function (contraction), while pairs (two losses) are enriched in protein maturation and transport between organelles (Additional file 9).

\section{Discussion}

We analyzed 61 animal genomes to reconstruct the evolutionary history of genes and chromosomes in the lineage leading to the ancestor of vertebrates, which then diversified in more than 60,000 species in the course of the following 450 million years. In contrast to previous studies which analyzed extant genomes, we first carefully reconstructed the ancestral Amniota genome to identify the signature of chromosome duplications more clearly. Our rationale is that the reconstructed ancestral Amniota genome should be devoid of the noise caused by the numerous rearrangements that took place during the following 326 million years of evolution. Indeed, the benefit of this approach can be seen when comparing the distribution of ohnologs in the ancestral Amniota genome versus their descendants in the human genome, for example in the chromosomes carrying the Hox clusters (Fig. 3c). The expected four-way association between duplicated chromosomes is striking in the Amniota reconstruction but blurred in the human genome. Statistical confidence and power are thus higher (Fig. 3d), enabling a higher resolution of chromosome events than was previously possible. In turn, this higher signal-to-noise ratio enables a straightforward reconstruction strategy of pre-duplication genomes, without requirements for complex statistical steps or algorithmic developments (reviewed in [30, 31]).

Progress was made on understanding early vertebrate genome evolution when Nakatani et al. [6] reconstructed a vertebrate pre-duplication ancestral genome by segmenting the human genome into conserved vertebrate linkage groups. These were compared with other vertebrate genomes, especially with medaka, using tunicate and sea urchin genes to define ohnologs. Using this strategy, the authors inferred a karyotype of the pre-1R vertebrate ancestor, but could not resolve inter-WGD chromosome fusions or fissions, leading to the conclusion that between 10 and 13 pre-1R chromosomes existed. Here, using the ancestral Amniota genome instead of the human genome, we reconstruct 17 chromosomes for the pre-1R genome and find evidence for 7 

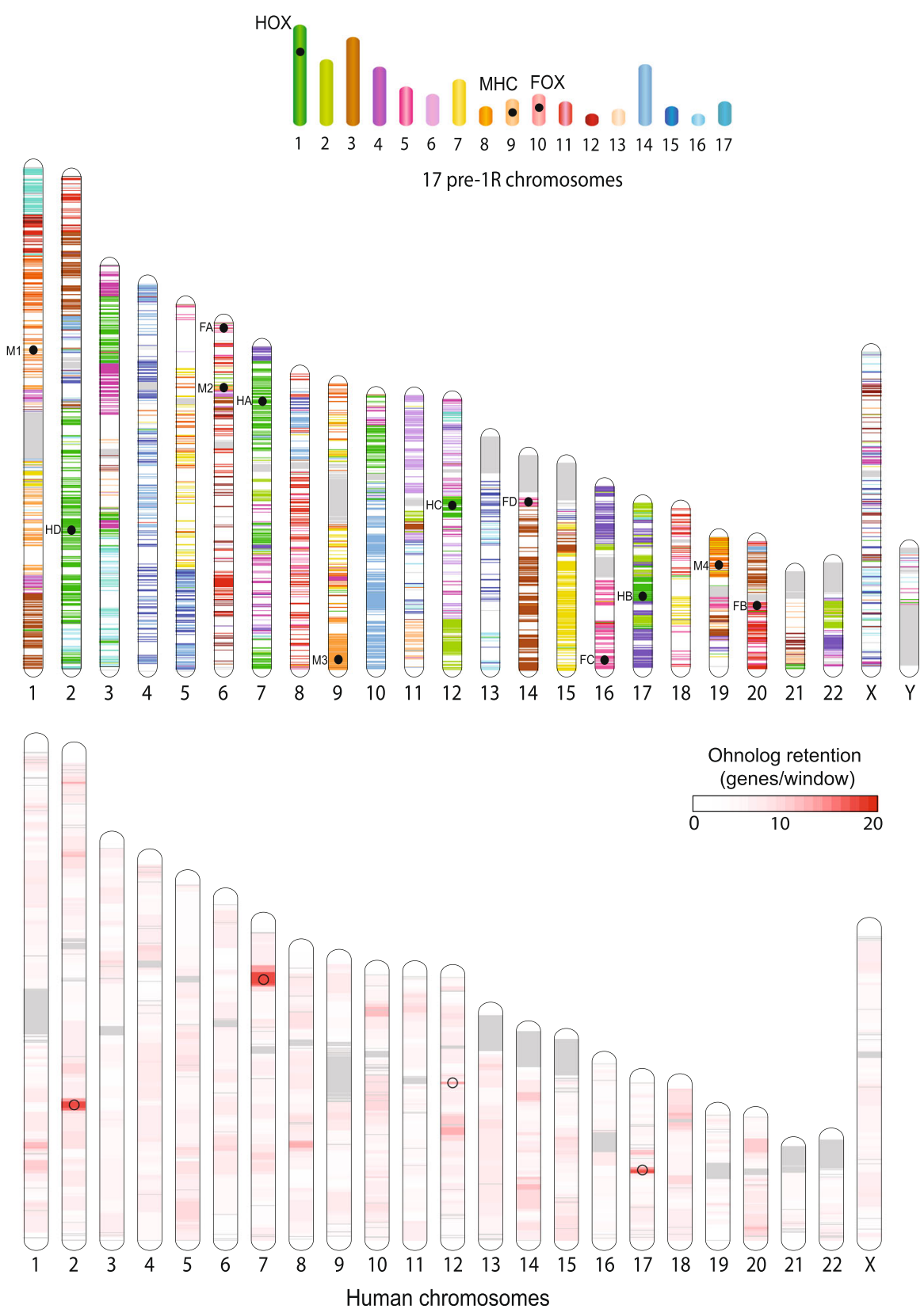

Fig. 6 Comparison between the pre-1R karyotype (top) composed of 17 chromosomes and the human karyotype (middle). The 8282 known human descendent genes of pre-1R genes are drawn at their position in the human genome with the color of their pre-1R ancestral chromosome. The position of 12 extant clusters (4 HOX, 4 FOX and $4 \mathrm{MHC}$ ) descending from a single clusters in pre-1R chromosomes are indicated by a black circle and a 2-character identifier (M1, M2, M3, M4 for MHC clusters, F1, F2, F3, F4 for FOX clusters, HA, HB, HC, HD for HOX clusters). A second human karyotype (bottom) shows, in a white-to-red scale, the number of ohnologs in windows of 50 genes positioned every 10 genes. Open circles denote the position of HOX clusters. Human chromosomes are drawn to scale, in Mb. Pre-1R chromosomes are drawn as in Fig. 5, in proportion to the number of genes assigned to each. The order of genes in pre-1R chromosomes being unknown, the positions of the 3 pre-1R gene clusters within their chromosome are arbitrary

fusions between the 2 WGDs but none for fissions. We therefore identify new chromosomes and find major differences between the two karyotypes (Additional file 1: Table S1). Our study provides an improved picture of ancestral vertebrate genome evolution in part because it is based on the reconstructed Amniota genome and also because it relied on information from more species and more recent genome annotations. For example the spotted gar genome [32] was more informative than the synteny with the medaka genome [19] because it lacks the numerous chromosome fusions that occurred before the teleost WGD [32]. Nakatani et al. also reconstructed an 
Osteichthyes post-2R genome corresponding here to the Euteleostomi ancestor, using a 2-of-3 rule relying on conservation between two of three genomes: the teleost pre-WGD, the chicken, and the vertebrate pre-1R genome [6]. Here however, our Euteleostomi ancestor is substantially different from this earlier study, since we describe an Euteleostomi genome of 50 chromosomes, not 31 . This difference mainly comes from fusions that we believe were incorrectly inferred from chicken-teleost genome comparisons, which were confounded by high rates of chromosome fusions in the lineage leading to the ancestral teleost [32]. Indeed, given that the ancestral teleost fish possessed only 13 chromosome, the 50 chromosomes inferred here in the ancestral Euteleostomi suggest that the rate of chromosomes fusions in the lineage leading to teleosts must have been more intense than previously thought, in the order of 37 fusions in 100-150 million years. Similarly, the ancestral eutherian karyotype probably consisted of 23 pairs [33], suggesting a consistent pattern of karyotype reduction by chromosome fusion after the $1 \mathrm{R}-2 \mathrm{R}$ whole genome duplications. These fusions in the teleost and mammalian lineages involved both macroand micro-chromosomes, probably explaining why these lineages do not possess micro-chromosomes any more.

We addressed several questions using the improved picture of ancestral vertebrate chromosomes described here. First, it should be noted that neither the reconstruction of the Amniota genome nor the establishment of ohnolog pairs described here make any assumption about the existence of two successive WGDs early in vertebrate evolution. Indeed, the criteria used to select ohnologs only rely on duplication dates and on local synteny, leaving open the possibility that segmental duplications, a single WGD or two WGDs, have occurred during early vertebrate genome evolution. But the fact that Amniota CARs readily associate to form tetrads when we used ohnologous genes as links (Fig. 3b), is a striking confirmation of the $1 \mathrm{R}-2 \mathrm{R}$ hypothesis. This scenario is in fact largely agreed upon today, but the debate was recently re-opened [16] after a reconstruction of the pre-1R vertebrate genome using the lamprey (Petromyzon marinus) genome sequence $[16,17]$ and the chicken genome. Lamprey is a Cyclostomata, a sister group to Gnathostomata [34] to which Amniotes belong, and both groups share the pre- $1 \mathrm{R}$ genome as common ancestor (Fig. 5). The lamprey genome is composed of almost 100 chromosomes, and its lineage separated from Gnathostomes soon after the last WGD. Redundant duplicate gene copies have therefore likely been lost largely independently in both lineages, leaving the possibility that a given Gnathostome (here chicken) chromosome would still display homologies to all lamprey chromosomes that derive from the same ancestral pre-1R chromosome [16]. Results of Smith et al. [16, 17] suggested that the ratio of ancestral pre- $1 \mathrm{R}$ chromosomes to chicken chromosomes was mostly $1: 2$ and less frequently 1:4 or even 1:3, thus supporting a single WGD ancestral to both lineages combined with additional large numbers of segmental duplications, at least in the lamprey lineage. However, when replicating the above study with our reconstructed ancestral Amniota genome instead of chicken, a clear majority of 1:4 patterns appears (Fig. 7 and Additional file 1), hence supporting the occurrence of two successive WGDs. This further emphasizes the benefit of using ancestral genome reconstructions as intermediates when investigating such ancient evolutionary events (about 450 million years before present). In addition, the clear 1:4 pattern is most parsimoniously explained if the Gnathostomes and the lamprey lineages share the $1 \mathrm{R}-2 \mathrm{R}$ duplications in their common ancestral history, which places the divergence of the Gnathostomes from the lamprey lineage after the $1 \mathrm{R}-2 \mathrm{R}$ duplications.

The reconstructed Amniota genome is not complete, as the 49 chromosomes only contain $80 \%$ of the 15,854 ancestral Amniota genes assigned to CARs. We note that although all chromosome tetrads corresponding to pre-1R chromosomes are complete (i.e., are composed of 4 CARs), the 49 reconstructed Amniota chromosomes display large differences in gene numbers: the largest contains 862 genes (chromosome 37) and the smallest only 16 genes (chromosome 49). This could reflect either a more intense process of gene inactivation and

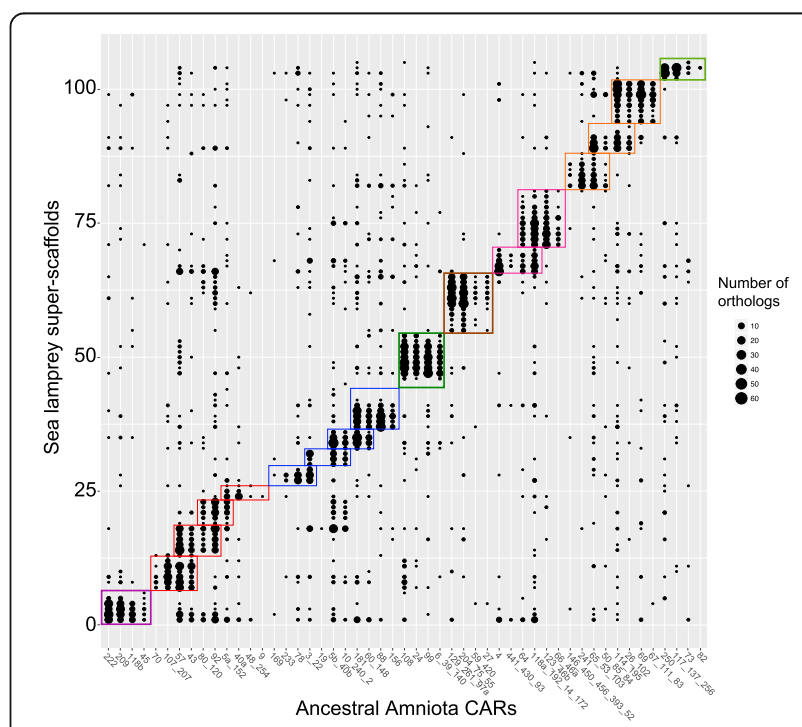

Fig. 7 Comparison of ancestral Amniota CARs with super-scaffolds of the lamprey (Petromyzon marinus) germline genome assembly [17]. Along the $X$-axis, Amniota CARs are grouped in the 17 tetrads (colored boxes) as shown in Fig. 3d. The order of the lamprey scaffolds on the $Y$-axis was designed as to cluster them according to orthology pattern against Amniota CARs. The size of each black circle is proportional to the number of orthologs between a given Amniota CAR (X-axis) and the corresponding lamprey scaffold (Y-axis) 
loss on chromosomes with fewer genes, or a more intense rate of rearrangement on those chromosomes, leading to greater difficulties in reconstructing them. Small chromosomes with few genes do not follow any noticeable pattern in their distribution among tetrads, which might have indicated systematic biases in gene deletion during rediploidization. We further examined if chromosomes could be paired within a tetrad, as expected if gene loss following the first WGD left a distinct pattern on the two ohnologous chromosomes, that would have propagated to the two duplicates resulting from the second WGD. Here again, such a pattern is not noticeable, which may indicate that the two WGDs took place in rapid succession, as suggested before [35], leaving little time for gene deletions (diploidization) to leave their imprint. More high-quality genome sequences from extant amniotes are required to improve the reconstruction of their ancestral genome and identify more subtle patterns left by the $1 \mathrm{R}-2 \mathrm{R}$. However, this current Amniota reconstruction and its ohnolog gene annotation already provide a solid foundation for new studies that should help resolve important questions, including complex phylogenetic histories [36].

An interesting question was raised during the analysis of the gar genome [32], when authors noticed a frequent 1:1 relationship between gar and chicken chromosomes, including micro-chromosomes. Micro-chromosomes are unusual because of their small sizes (usually below $20 \mathrm{Mb}$ in chicken), their high GC content, high recombination rates, and high gene density. The gar-chicken comparison suggests that micro-chromosomes are ancestral features in Euteleostomi, which in turn raises the question of their origin through the $1 \mathrm{R}-2 \mathrm{R}$ duplications. Twelve gar and chicken micro-chromosomes are homologous and can parsimoniously be considered ancestral to Euteleostomi (Additional file 1: Table S2). They are distributed in 7 to 11 tetrads (some Euteleostomi micro-chromosomes originate from two tetrads), each of these tetrads containing both micro- and macro-chromosomes (Fig. 5). This leaves open the question of the timing of their formation: before the $1 \mathrm{R}$, between the $1 \mathrm{R}$ and $2 \mathrm{R}$, or immediately after the $2 \mathrm{R}$.

The pre-2R karyotype and its evolution described here provide a framework to study the impact of the $1 R-2 R$ in extant vertebrate genomes, especially via the set of phylogenetically consistent ohnolog families that underlie the analysis. For example, previous studies have provided alternative hypotheses to explain a possible biased retention of ohnologs, including a requirement for stoichiometric balance of proteins in complexes $[8,37]$ or the preferential retention of genes involved in diseases owing to their dominant negative consequence on fitness if deleted from the genome [12, 38]. Ohnologs have also been shown to be enriched in developmental genes [39] and regulatory function [11]. A precise mechanism to explain biased ohnolog retention, if any, remains to be established. This may be because these studies are based on extant genes copies, and 500 million years of evolution have elapsed since the 1R-2R WGDs. Using the human descendants of ohnologous Amniota genes, we show that a striking pattern of biological functions are preferentially retained in ohnologous families that were subject to two gene losses, one gene loss or remained as a quartet of genes. Such distinctive and specific distribution of functions between the three types of ohnolog families raises the question of strong functional constraints governing early rates of ohnolog retention and loss. It is possible that these patterns reflect the functions and anatomical parts (central nervous system and muscles) most targeted by selection during early vertebrate evolution, when gene redundancy was maximum and provided a template for evolutionary innovations, leading to increased organismal complexity. This further illustrates the benefit of comparative genomics based on reconstructed ancestral genomes.

\section{Conclusion}

Biology is a historical science, but this historical dimension is often difficult to acknowledge because the genomic records required to document ancestral states are missing. In practical terms this lack of information hinders our ability to integrate conclusions made across different living organisms, and to draw all the benefits from comparative genomics. Here, the detailed reconstruction of the early history of the vertebrate genome is a step towards a better understanding of the founding events at the origin of living mammals, birds, reptiles, amphibians and fish. It provides a new perspective, that of a common reference, to study the evolution of these extant animals.

\section{Methods \\ Phylogenetic gene trees}

The complete set of 20,285 phylogenetic gene trees built by the Ensembl Compara pipeline (Ensembl version 69) [40] were downloaded using the Ensembl API. Duplication nodes were edited when their consistency score was below 0.3 , as described in [41]. These phylogenetic gene trees were used in this study to (i) identify ancestral genes and orthologous gene relationships when reconstructing the Amniota ancestral genome, (ii) identify ohnologous gene relationships when constructing the "A" list of ohnologous gene pairs, and (iii) identify the ancestral and extant gene copies of genes from the original $\mathrm{B}$ and $\mathrm{C}$ lists of ohnologs and update the human-CLG relationships from reference [5] (all built using other Ensembl versions). 


\section{Reconstruction of the Amniota ancestral genome}

The ancestral Amniota genome was reconstructed using the AGORA (Algorithm for Gene Order Reconstruction in Ancestors) [18] (https://github.com/DyogenIBENS), which is routinely used to reconstruct ancestral gene order presented in Genomicus since Ensembl release 53. The Amniota reconstruction used here is available for download on the $\mathrm{ftp}$ site of the Genomicus webserver [23] (ftp:// ftp.biologie.ens.fr/pub/dyogen/genomicus/69.10/ and [42]). AGORA takes as input the gene orders, gene orientations, and gene trees from 61 extant metazoan genomes available in release 69 of Ensembl (2012/11/15). The 61 species are listed in the Additional file 1: Table S4 and include 40 mammals, 3 birds, 1 reptile, 1 turtle, 1 amphibian, 1 coelacanth, 1 lamprey, and 8 teleost fish among vertebrates, and 2 Cionas, 1 fruit fly and 1 nematode. The corresponding species tree can be found at http://www.genomicus.biologie.ens.fr/genomicus-69.01/data/SpeciesTree.pdf

Briefly, AGORA is a graph-based parsimony method: it builds an adjacency graph where ancestral genes are vertices (nodes) and ancestral adjacencies are edges (links) weighted by the frequency of their conservation. This frequency is measured by comparing pairs of genomes descending independently from the Amniota ancestor (one Mammalia and one Sauropsida) or one descendant and one outgroup, and counting how many times a given ancestral gene adjacency is conserved between the two extant genomes being compared. The graph is then linearized along the edges of maximal weight to produce Contiguous Ancestral Regions (CARs). An AGORA reconstruction of the Amniota genome based on Ensembl version 84 (2016/03/15) was also used for comparison (Table 1).

\section{Identification of ohnolog gene pairs in the Amniota ancestor}

Human ohnolog gene pairs from [8] based on Ensembl 52 data were assigned to their Ensembl version 69 gene trees using Ensembl gene IDs. Their Amniota ancestral genes were used to build list B of ohnolog gene pairs. Similarly, we downloaded three lists of ohnolog gene pairs from http://ohnologs.curie.fr/ described in [9], each list corresponding to a different degree of confidence level. A non-redundant list of Amniota ancestral genes was identified in Ensembl version 69 by sequentially identifying the Amniota ancestral genes of these ohnologs (data downloaded on October 27, 2014). We built list A by identifying ohnolog pairs directly in the Amniota reconstructed genome starting from ancestral genes that were duplicated between the Chordata and the Euteleostomi ancestors: these candidate pairs were considered ohnologs if another candidate pair from a different gene tree could be found on the same pair of CARs at a distance $\leq N$ genes (to account for massive duplicate loss after a WGD): the parameter $N$ was made vary to optimize both sensitivity and specificity and fixed to 45 genes. A pair of ohnologs was allowed to occur between genes of the same CAR if they were located at a distance of $2 N$ genes (90 genes, to account for possible rearrangements on the Amniota lineage), which added only 44 pairs. An integrated, high-quality list of ohnolog gene pairs was built from these five lists. Starting from their intersection consisting of 1273 pairs, we built disjoint graphs of ohnologs connected by ohnologous relationships. Replacing genes by the CARs they belong to, these graphs never involved more than four CARs of at least ten genes. To this core list, we sequentially added lists of pairs present in several or only one of the five initial lists, removing at each step the newly added pairs that would build ohnolog networks involving more than four CARs (Additional file 2: Table S5). The order in which these added lists were considered was established using the levels of confidence of the lists $C$, the number of lists where the pairs were found and the 1R-2R-compatibility criterion: a list was of better quality if it created fewer ohnolog networks of more than four CARs (per added pair) when added to the current validated list. Two properties of list A were maintained along the process: (1) the two ohnologs of a pair were allowed to be located on the same CAR only if they were $\geq$ 90 genes away from each other; (2) the two ohnologs of the pair had to belong to the same Ensembl gene tree.

\section{Identification of ohnolog CARs in the Amniota ancestor}

Given the distribution of ohnologs on the Amniota CARs, a proportion test was performed (prop.test function in R) between each pair of CARs to estimate if the corresponding CARs shared more ohnolog pairs than expected by chance. Bonferroni adjusted $p$ values of 0.05 or less were considered significant. However, $p$ values obtained without the Bonferroni correction were also examined if CARs were included in a tetrad with a least one significant Bonferroni-adjusted $p$ value.

\section{Attribution of ancestral Olfactores genes to the 17 pre-1R chromosomes}

Phylogenetic gene trees from version 69 of Ensembl were analyzed to identify 10,093 nodes (genes) in the ancestral Olfactores genome, which were assigned to the 17 pre- $1 \mathrm{R}$ tetrads according to the distribution of their descendent ancestral Amniota genes in the Amniota CARs used to build ohnologous tetrads. In the simple case of a single disjoint tetrad, all ancestral Olfactores genes with descendants exclusively in the 4 CARs of a tetrad were included in the corresponding pre-1R chromosome. Ancestral Olfactores genes with descendants in more than one tetrad were considered ambiguous and excluded. In the more complex cases of adjacent tetrads, the same principle was applied but when CARs belonged to two adjacent tetrads, only 
ohnolog genes were retained. In order to maximize the number of ancestral genes in the pre-1R chromosomes, we used all possible small CARs ( $\leq 50$ genes) that could be assembled to the CARs of the tetrads, respecting all criteria of the 23 previous assemblies (Additional file 10).

\section{Human genes derived from ancestral chordate linkage groups (CLGs)}

The coordinates of the 120 human chromosome segments from Tables S1 and S14 in reference [7] were updated from the hg18 to hg19 version of the human genome using the UCSC liftOver utility. Twenty-two segments were not converted by liftOver and were further fragmented in 100 sub-segments of equal size, which were mapped again to hg19 in order to recover as many genes as possible (Additional file 1: Figure S15).

\section{Gene Ontology (GO) analysis}

The 2973 Amniota ohnolog families each contain from 2 to 11 ohnologs, depending on gene loss and duplications that occurred between the $1 \mathrm{R}$ and the ancestor of amniotes. To perform the GO analysis, we identified families containing a maximum of 4 ohnologs and where each ohnolog was located on a different CAR (1516 pairs, 502 triplets, 172 quartets). Ensembl gene trees were then used to identify human descendants of each ohnolog, if it exists. Lists of human genes were used on the Gorilla server [43], comparing each ohnolog list against the rest of the genome. After selecting GO terms enriched with an FDR $<10^{-5}$, terms were ranked by decreasing enrichment fold and the first $30 \mathrm{GO}$ terms were analyzed and reported in Additional file 9.

\section{Additional files}

Additional file 1: Additional methods, tables and figures, and a full description of all additional files. (DOCX $8000 \mathrm{~kb}$ )

Additional file 2: Describes the incremental construction of the list of ohnologs. (XLSX $18 \mathrm{~kb}$ )

Additional file 3: List of 8184 ancestral Amniota ohnolog gene names and their human descendent gene names (Ensembl gene ID). (TXT $336 \mathrm{~kb}$ )

Additional file 4: List of the 7441 pairs of ancestral Amniota ohnolog genes constructed in this work. (TXT $371 \mathrm{~kb}$ )

Additional file 5: List of the ohnolog families (ohnolog genes linked by pairs) from the list of ancestral Amniota ohnolog pairs in Additional file 4. (TXT $200 \mathrm{~kb}$ )

Additional file 6: Summarizes the evidence supporting tetrads. (XLSX $78 \mathrm{~kb})$

Additional file 7: Describes the comparison of the 17 tetrads to the 17 CLG of ref. [7]. (XLSX $83 \mathrm{~kb})$

Additional file 8: List of the composition of the 17 ancestral vertebrate chromosomes in terms of Olfactores ancestral genes (Ensembl gene ID). (TXT $459 \mathrm{~kb}$ )

Additional file 9: Results of the Gene Ontology enrichment analysis of human descendant of pairs, triplets, and quartets of ancestral Amniota ohnologs. (XLSX $57 \mathrm{~kb}$ )
Additional file 10: Summarizes the evidence supporting the addition of small CARs to tetrads. (XLSX $45 \mathrm{~kb}$ )

\section{Acknowledgements}

We wish to thank Yann Guiguen for commenting on the manuscript, Pierre Vincens for the coordination of computing resources, and Lucas Tittmann for the assistance with statistics.

Funding

This work was supported by grants from the French Government and implemented by ANR (ANR-10-BINF-01-03 Ancestrome, ANR-10-LABX-54 MEMOLIFE and ANR-10-IDEX-0001-02 PSL* Research University).

Availability of data and materials

All data generated during this study are included in this published article. In addition, a dedicated Genomicus server (http://

www.genomicus.biologie.ens.fr/genomicus-69.10/) [44] provides a graphical access to the data available as plain text at https://www.ebi.ac.uk/biostudies/ studies/S-BSST199 [42].

\section{Authors' contributions}

CS designed the experiments, performed the analyses, and helped write the manuscript. AL performed the ancestral Amniota genome reconstruction, the $\mathrm{GO}$ analysis, contributed to the comparison with the lamprey genome and built the Genomicus website. C. Bon built the ohnolog list A. C Berthelot helped write the manuscript. HRC designed the study, designed the experiments, performed the analyses, and wrote the manuscript. All authors read and approved the final manuscript.

Ethics approval and consent to participate

Not applicable

\section{Consent for publication}

Not applicable

\section{Competing interests}

The authors declare that they have no competing interests.

\section{Publisher's Note}

Springer Nature remains neutral with regard to jurisdictional claims in published maps and institutional affiliations.

\section{Author details}

'Institut de Biologie de l'Ecole Normale Supérieure (IBENS), Ecole Normale Supérieure, CNRS, INSERM, PSL Research University, 75005 Paris, France. ${ }^{2}$ Present Address: Laboratoire Éco-Anthropologie et Ethnobiologie, UMR 7206 CNRS - Muséum National d'Histoire Naturelle, Université Paris Diderot, Sorbonne Paris Cité, F-75016 Paris, France.

Received: 9 February 2018 Accepted: 4 October 2018

Published online: 17 October 2018

References

1. Innan $\mathrm{H}$, Kondrashov F. The evolution of gene duplications: classifying and distinguishing between models. Nat Rev Genet. 2010;11:97-108.

2. Conrad B, Antonorakis, SE. Gene duplication: a drive for phenotypic diversity and cause of human disease. Annu Rev Genomics Hum Genet. 2007:8:17-35.

3. Van de Peer $Y$, Maere $S$, Meyer A. The evolutionary significance of ancient genome duplications. Nat Rev Genet. 2009;10:725-32.

4. Ohno S. Evolution by gene duplication. London: Allen and Unwin; 1970.

5. Dehal P, Boore JL. Two rounds of whole genome duplication in the ancestral vertebrate. PLoS Biol. 2005:3:e314.

6. Nakatani $Y$, Takeda H, Kohara Y, Morishita S. Reconstruction of the vertebrate ancestral genome reveals dynamic genome reorganization in early vertebrates. Genome Res. 2007;17:1254-65.

7. Putnam NH, Butts $T$, Ferrier DEK, Furlong RF, Hellsten U, Kawashima T, Robinson-Rechavi M, Shoguchi E, Terry A, Yu J-K, et al. The amphioxus genome and the evolution of the chordate karyotype. Nature. 2008;453: 1064-71. 
8. Makino T, McLysaght A. Ohnologs in the human genome are dosage balanced and frequently associated with disease. Proc Natl Acad Sci. 2010; 107:9270-4

9. Singh PP, Arora J, Isambert H. Identification of ohnolog genes originating from whole genome duplication in early vertebrates, based on synteny comparison across multiple genomes. PLoS Comput Biol. 2015;11:e1004394.

10. Amores A, Force A, Yan YL, Joly L, Amemiya C, Fritz A, Ho RK, Langeland J, Prince $V$, Wang $Y L$, et al. Zebrafish hox clusters and vertebrate genome evolution. Science. 1998;282:1711-4.

11. Blomme T, Vandepoele K, De Bodt S, Simillion C, Maere S, Van de Peer Y: The gain and loss of genes during 600 million years of vertebrate evolution. Genome Biol 2006, 7:R43.

12. Singh PP, Affeldt S, Cascone I, Selimoglu R, Camonis J, Isambert H. On the expansion of "dangerous" gene repertoires by whole-genome duplications in early vertebrates. Cell Rep. 2012;2:1387-98.

13. McLysaght A, Makino T, Grayton HM, Tropeano M, Mitchell KJ, Vassos E, Collier DA. Ohnologs are overrepresented in pathogenic copy number mutations. Proc Natl Acad Sci U S A. 2014;111:361-6.

14. Rice AM, McLysaght A. Dosage sensitivity is a major determinant of human copy number variant pathogenicity. Nat Commun. 2017:8:14366.

15. Makino T, McLysaght A, Kawata M. Genome-wide deserts for copy number variation in vertebrates. Nat Commun. 2013;4:2283.

16. Smith JJ, Keinath MC. The sea lamprey meiotic map improves resolution of ancient vertebrate genome duplications. Genome Res. 2015;25:1081-90.

17. Smith JJ, Timoshevskaya N, Ye C, Holt C, Keinath MC, Parker HJ, Cook ME, Hess JE, Narum SR, Lamanna F, et al. The sea lamprey germline genome provides insights into programmed genome rearrangement and vertebrate evolution. Nat Genet. 2018;50:270-7.

18. Berthelot C, Muffato M, Abecassis J, Roest Crollius H. The 3D organization of chromatin explains evolutionary fragile genomic regions. Cell Rep. 2015;10:1913-24.

19. Kasahara M, Naruse K, Sasaki S, Nakatani Y, Qu W, Ahsan B, Yamada T, Nagayasu Y, Doi K, Kasai Y, et al. The medaka draft genome and insights into vertebrate genome evolution. Nature. 2007:447:714-9.

20. Jaillon O, Aury J-M, Brunet F, Petit J-L, Stange-Thomann N, Mauceli E, Bouneau L, Fischer C, Ozouf-Costaz C, Bernot A, et al. Genome duplication in the teleost fish Tetraodon nigroviridis reveals the early vertebrate protokaryotype. Nature. 2004;431:946-57.

21. Kellis M, Birren BW, Lander ES. Proof and evolutionary analysis of ancient genome duplication in the yeast Saccharomyces cerevisiae. Nature. 2004; 428:617-24.

22. Louis A, Roest Crollius H, Robinson-Rechavi M. How much does the amphioxus genome represent the ancestor of chordates? Brief Funct Genomics. 2012;11:89-95.

23. Nguyen NTT, Vincens P, Roest Crollius H, Louis A. Genomicus 2018: karyotype evolutionary trees and on-the-fly synteny computing. Nucleic Acids Res. 2017:46:D816-D822.

24. Krumlauf R. Hox genes in vertebrate development. Cell. 1994;78:191-201.

25. Danchin EG, Pontarotti P. Towards the reconstruction of the bilaterian ancestral pre-MHC region. Trends Genet. 2004;20:587-91.

26. Wotton KR, Shimeld SM. Comparative genomics of vertebrate Fox cluster loci. BMC Genomics. 2006;7:271.

27. Morison IM, Reeve AE. A catalogue of imprinted genes and parent-of-origin effects in humans and animals. Hum Mol Genet. 1998:7:1599-609.

28. Dunzinger U, Haaf T, Zechner U. Conserved synteny of mammalian imprinted genes in chicken, frog, and fish genomes. Cytogenet Genome Res. 2007;117:78-85

29. Renfree MB, Hore TA, Shaw G, Graves JA, Pask AJ. Evolution of genomic imprinting: insights from marsupials and monotremes. Annu Rev Genomics Hum Genet. 2009:10:241-62.

30. Muffato $M$, Roest Crollius H. Paleogenomics in vertebrates, or the recovery of lost genomes from the mist of time. Bioessays. 2008;30:122-34.

31. Nakatani $Y$, McLysaght A. Genomes as documents of evolutionary history: a probabilistic macrosynteny model for the reconstruction of ancestral genomes. Bioinformatics. 2017;33:1369-78.

32. Braasch I, Gehrke AR, Smith JJ, Kawasaki K, Manousaki T, Pasquier J, Amores A, Desvignes T, Batzel P, Catchen J, et al. The spotted gar genome illuminates vertebrate evolution and facilitates human-teleost comparisons. Nat Genet. 2016:48:427-37.
33. Ferguson-Smith MA, Trifonov V. Mammalian karyotype evolution. Nat Rev Genet. 2007:8:950-62.

34. Heimberg AM, Cowper-Sal-lari R, Semon M, Donoghue PC, Peterson KJ. microRNAs reveal the interrelationships of hagfish, lampreys, and gnathostomes and the nature of the ancestral vertebrate. Proc Natl Acad Sci U S A. 2010;107:19379-83.

35. Furlong RF, Holland PW. Were vertebrates octoploid? Philos Trans R Soc Lond Ser B Biol Sci. 2002;357:531-44.

36. Lynch VJ, Wagner GP. Multiple chromosomal rearrangements structured the ancestral vertebrate Hox-bearing protochromosomes. PLoS Genet. 2009;5: e1000349.

37. Makino T, Hokamp K, Mclysaght A. The complex relationship of gene duplication and essentiality. Trends Genet. 2009;25:152-5.

38. Singh PP, Affeldt S, Malaguti G, Isambert H. Human dominant disease genes are enriched in paralogs originating from whole genome duplication. PLoS Comput Biol. 2014;10:e1003754.

39. Brunet FG, Roest Crollius H, Paris M, Aury J-M, Gibert P, Jaillon $O$ Laudet $V$, Robinson-Rechavi M. Gene loss and evolutionary rates following whole-genome duplication in teleost fishes. Mol Biol Evol. 2006;23:1808-16.

40. Vilella AJ, Severin J, Ureta-Vidal A, Heng L, Durbin R, Birney E. EnsemblCompara GeneTrees: complete, duplication-aware phylogenetic trees in vertebrates. Genome Res. 2009;19:327-35.

41. Peres A, Roe st Crollius H. Improving duplicated nodes position in vertebrate gene trees. BMC Bioinformatics. 2015;16(Suppl. 3):A9.

42. Sacerdot C, Louis A, Bon C, Berthelot C, Roest Crollius H: Chromosome evolution at the origin of the ancestral vertebrate genome - dataset. https://http://www.ebi.ac.uk/biostudies/studies/S-BSST199; 2018.

43. Eden E, Navon R, Steinfeld I, Lipson D, Yakhini Z. GOrilla: a tool for discovery and visualization of enriched $\mathrm{GO}$ terms in ranked gene lists. BMC Bioinformatics. 2009:10:48

44. Louis A, Sacerdot C, Roest Crollius H: Chromosome evolution at the origin of the ancestral vertebrate genome - Genomicus server. http://www. genomicus.biologie.ens.fr/genomicus-69.10/. 2018.

45. Krzywinski M, Schein J, Birol I, Connors J, Gascoyne R, Horsman D, Jones SJ, Marra MA. Circos: an information aesthetic for comparative genomics. Genome Res. 2009:19:1639-45.

\section{Ready to submit your research? Choose BMC and benefit from:}

- fast, convenient online submission

- thorough peer review by experienced researchers in your field

- rapid publication on acceptance

- support for research data, including large and complex data types

- gold Open Access which fosters wider collaboration and increased citations

- maximum visibility for your research: over $100 \mathrm{M}$ website views per year

At $\mathrm{BMC}$, research is always in progress.

Learn more biomedcentral.com/submission 